EPJ Web of Conferences 69, 00007 (2014)

DOI: $10.1051 /$ epjconf / 20146900007

(C) Owned by the authors, published by EDP Sciences, 2014

\title{
Coupled channels optical model potential for rare earth nuclei
}

\author{
M. Herman ${ }^{1, a}$, G. P. A. Nobre ${ }^{1, b}$, A. Palumbo ${ }^{1}$, F. S. Dietrich ${ }^{2}$, D. Brown ${ }^{1}$, and S. Hoblit ${ }^{1}$ \\ ${ }^{1}$ National Nuclear Data Center, Brookhaven National Laboratory, Upton, NY 11973-5000, USA \\ ${ }^{2}$ P.O. Box 30423, Walnut Creek, CA, 94598, USA
}

\begin{abstract}
The global spherical optical model by Koning and Delaroche is generalized to enable its use in coupled-channel calculations on well deformed nuclei in the rare-earth region. The generalization consists in adding the coupling of the ground state rotational band, deforming the potential by introducing appropriate quadrupole and hexadecupole deformations and correcting the optical model potential radius to preserve the volume integral of the spherical optical potential. We choose isotopes of three rare-earth elements (W, Ho, Gd), which are known to be nearly perfect rotors, to perform a consistency test of our conjecture on integrated cross sections as well as on angular distributions for elastic and inelastic neutron scattering. The only additional input are experimentally determined deformations, which we employ without any adjustments. The results are clearly superior compared to the spherical optical model calculations with dramatic improvement at low incident energies.
\end{abstract}

\section{Introduction}

The coupled-channel theory is a natural way of accounting for nonelastic channels, in particular those arising from collective excitations. Proper treatment of such excitations is often essential to the accurate description of reaction experimental data. Optical potentials (OP) needed for coupled-channels calculations are normally obtained through proper parametrization and parameter fitting in order to reproduce experimental data sets for a specific nucleus. Such phenomenological OP's might achieve very good agreement with experimental data, as they were specifically adjusted to do so, but do not lend themselves to extrapolation to other nuclei, unless there are explicit measurements that allow to readjust OP individually. Therefore, the predictive power of the coupled-channels method is hampered by the lack of reliable OP for the nuclei with no or scarce experimental data. On the other hand, there are quite reliable spherical OP's applicable to spherical or slightly-deformed nuclei. Therefore, developing a method capable of employing such well-tested global OP's in the particular case of deformed nuclei would be desirable.

Inspired by the recent work by Dietrich et al., substantiating the validity of the adiabatic assumption in coupled-channel calculations, we explore the possibility of generalizing (deforming) a global spherical optical model potential to make it usable in coupled-channel calculations on statically deformed nuclei.

\footnotetext{
a e-mail: mwherman@bnl.gov

be-mail: gnobre@bnl.gov
}

This is an Open Access article distributed under the terms of the Creative Commons Attribution License 2.0, which permits unrestricted use, distribution, and reproduction in any medium, provided the original work is properly cited. 
Optical potentials (OP) have been widely used to describe nuclear reaction data by implicitly accounting for the effects of excitation of internal degrees of freedom and other nonelastic processes. An OP is called global when this fitting process is consistently done for a variety of nuclides.

There are global spherical OP's that have been fit to nuclei below and above the region of statically deformed rare-earth nuclei, but these potentials have been viewed as inappropriate for use in coupledchannels calculations, since they do not account for the loss of flux through the explicitly included inelastic channels. On the other hand, a recent paper [1] shows that scattering from rare earth and actinide nuclei is very near the adiabatic (frozen nucleus) limit, which suggests that the loss of flux to rotational excitations might be unimportant. In this paper we test this idea by performing coupled channels calculations with a global spherical optical potential by deforming the nuclear radii but making no further adjustments. We note an alternative approach (Kuneida et al. [2]), which has attempted to unify scattering from spherical and deformed nuclei by considering all nuclei as statically deformed, regardless of their actual deformation.

This work corresponds to a preliminary attempt to extend the approach initially presented in Ref. [3], focusing on angular distributions for the cases of neutron scattered by Gd, Ho, and W nuclei.

\section{Adiabatic model for rare-earths}

Due to the high moment of inertia and consequent low excitation energies of the ground-state band members of the statically deformed nuclei in the rare-earth region, the deformed nuclear configuration may be regarded as "frozen" during the scattering. This means that all the internal degrees of freedom not associated with the strong deformation may assumed to be accounted for by a spherical optical potential that describes well the nuclei in the neighboring region, in an adiabatic approach. Therefore, the only channels that need to be treated explicitly (e. g., through coupled channels methods) are the ones arising from the static deformation.

The spherical OP that was deformed in our coupled-channel calculations was the global KoningDelaroche (KD) [4], unmodified except for a small change in the radius parameters to ensure volume conservation when the nucleus is deformed. Since the KD potential describes scattering from nuclei both above and below the deformed rare earth region very well, we make the assumption that the imaginary potential adequately describes the internal nuclear excitations in the rare earths also. This picture is consistent with the adiabatic approximation. The coupled channels calculations account for the external (rotational) excitations of the target. These assumptions are tested in the calculations shown in this paper.

The process of deforming a spherical OP to explicitly consider collective excitations within the coupled channels framework is done in the standard way by replacing the radius parameter $R$ in each Woods-Saxon form factor with the angle dependent expression:

$$
R(\theta)=R_{0}\left(1+\sum_{\lambda} \beta_{\lambda} Y_{\lambda 0}(\theta)\right)
$$

where $R_{0}$ is the undeformed radius of the nucleus, and $\beta_{\lambda}$ and $Y_{\lambda 0}(\theta)$ are the deformation parameter and spherical harmonic for the multipole $\lambda$, as seen in Ref. [5], for example. The deformed form factor obtained from Eq. 1 is then expanded in Legendre polynomials numerically.

We use in our calculations the EMPIRE reaction code $[6,7]$, in which the direct reaction part is calculated by the code Ecis $[8,9]$. In order to test our model we perform coupled-channel calculations, coupling the ground state rotational band, for neutron-incident reactions on selected rare-earth nuclei, namely ${ }^{152,154} \mathrm{Sm},{ }^{153} \mathrm{Eu},{ }^{155,156,157,158,160} \mathrm{Gd},{ }^{159} \mathrm{~Tb},{ }^{162,163,164} \mathrm{Dy},{ }^{165} \mathrm{Ho},{ }^{166,167,168,170} \mathrm{Er},{ }^{169} \mathrm{Tm}$, ${ }^{171,172,173,174,176} \mathrm{Yb},{ }^{175,176} \mathrm{Lu},{ }^{177,178,179,180} \mathrm{Hf},{ }^{181} \mathrm{Ta}$, and ${ }^{182,183,184,186} \mathrm{~W}$. All those nuclides have at least 


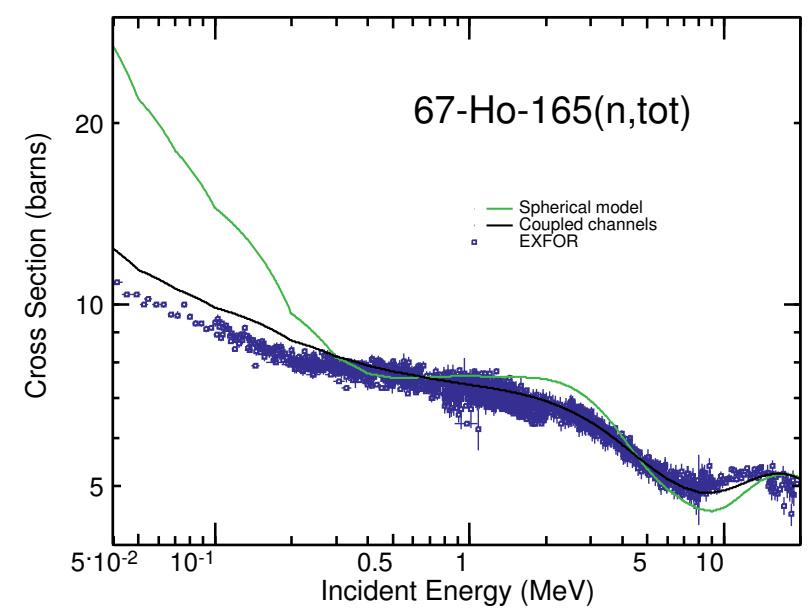

Figure 1. Total cross section for neutron-induced reactions on ${ }^{165} \mathrm{Ho}$. The black curve corresponds to coupledchannel calculations within our model, while the green curve indicates, for comparison purposes, the result from a spherical model calculation. The experimental data were taken from EXFOR [11].

90 neutrons, indicating static deformation, therefore making them suitable candidates for interpolation through the adiabatic limit. We then compared, as an initial test, the obtained coupled-channel results for total cross sections with plain spherical calculations with the undeformed KD optical potential. In this initial step, only quadrupole deformations were considered, having their values taken from the compilation of experimental values from Raman et al. [10]. The overall result is a dramatic improvement in the agreement with experimental data, in particular in the lower neutron-incident energies. Fig. 1 clearly illustrates the very good description of the observed total cross section in the case of ${ }^{165}$ Ho, obtained through our coupled-channel model.

In carrying out the calculations, it is important to couple a sufficient number of rotational states to achieve convergence and we have carried out tests to ensure this. Such an analysis is shown in Ref. [12], where it is also demonstrated that this convergence can be energy-dependent.

\subsection{Compound-nucleus observables}

After the initial success in describing direct-reaction quantities, such as total cross sections, we analyzed the model predictions for observables that depend also on the compound-nucleus decay. The models adopted to describe the emissions from the compound nucleus were basically default options in EMPIRE code, which means a standard Hauser-Feshbach model with properly parametrized Enhanced Generalized Superfluid Model (EGSM) level densities [13], modified Lorentzian (version 1) $\gamma$-ray strength functions [14-16], width fluctuation corrections up to $3 \mathrm{MeV}$ in terms of the HRTW approach [17, 18], and with transmission coefficients for the inelastic outgoing channels also calculated within the coupled-channel approach. Pre-equilibrium emission was calculated within the exciton model [19], as based on the solution of the master equation [20] in the form proposed by Cline [21] and Ribansky [22] (using Pcross code [6,7]) with the mean free path multiplier set to 1.5.

In Fig. 2, as an example, we compare with experimental data the angle-integrated elastic cross sections for incident neutrons on ${ }^{165} \mathrm{Ho}$ and ${ }^{156} \mathrm{Gd}$ obtained by our coupled-channel calculations. Even though there are not as many data available as in the case of total cross sections (Fig. 1), the low-energy 


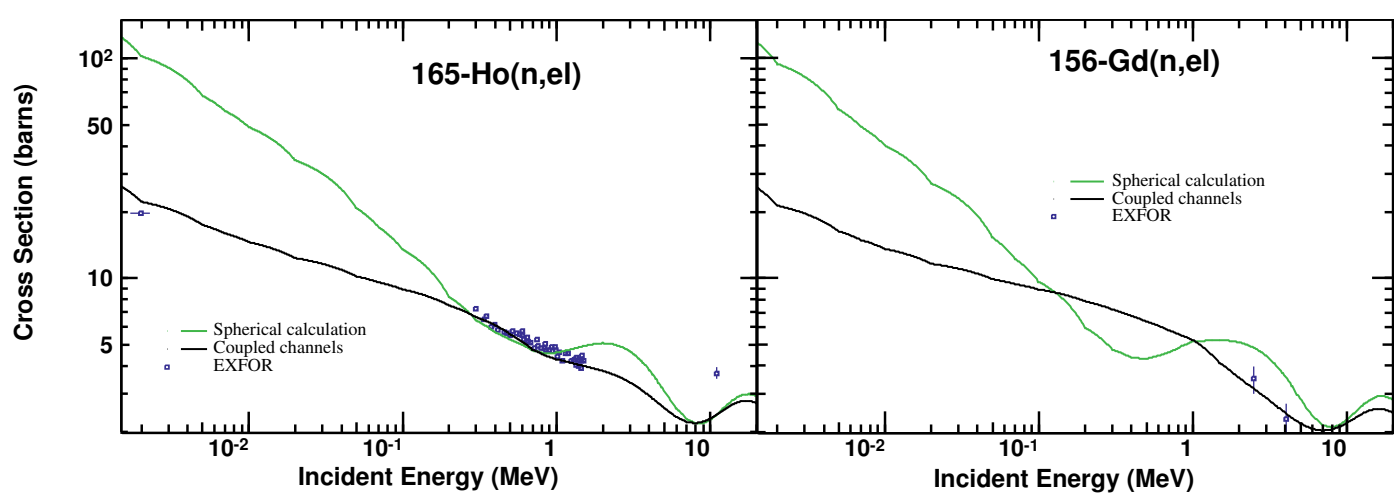

Figure 2. Angle-integrated elastic cross sections for the case of ${ }^{165} \mathrm{Ho}$ (left panel) and ${ }^{156} \mathrm{Gd}$ (right panel) targets. Black curves correspond to predictions by our coupled-channel model while green curves were obtained by spherical model calculations. The experimental data were taken from EXFOR [11].

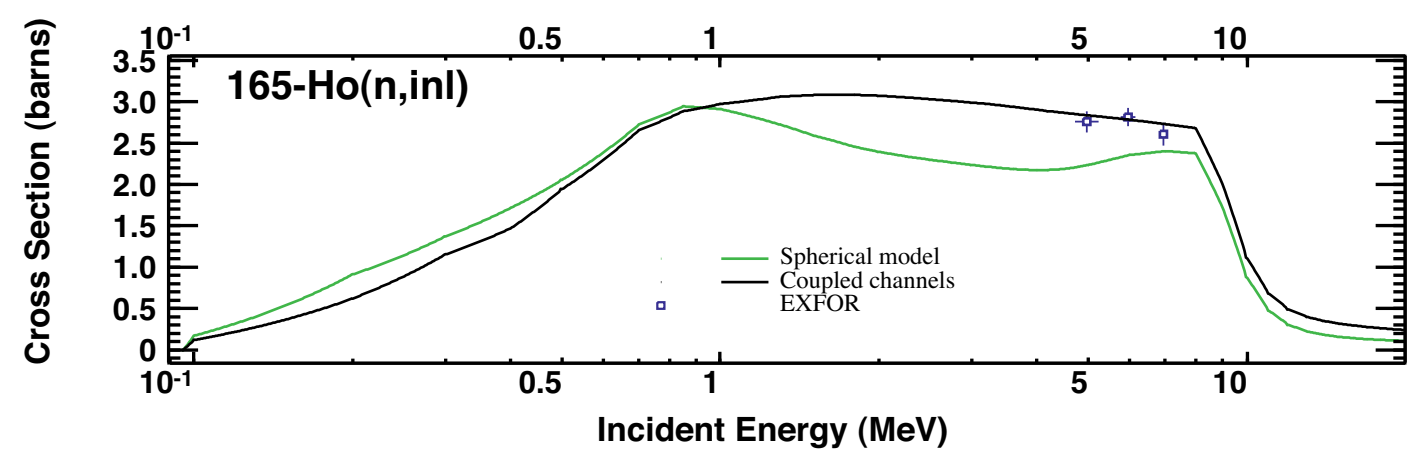

Figure 3. Angle-integrated inelastic cross sections for ${ }^{165} \mathrm{Ho}$. Black curves correspond to predictions by our coupled-channel model while green curves were obtained by spherical model calculations. The experimental data were taken from EXFOR [11].

point ( $\sim 3 \mathrm{keV}$ ) for ${ }^{165} \mathrm{Ho}$ (Fig. 2, left panel) and the few high energy points ( $22 \mathrm{MeV}$ ) for ${ }^{156} \mathrm{Gd}$ (Fig. 2, right panel) indicate again a very good agreement between our coupled channels model and the experimental data (in contrast with the spherical-model calculations).

Fig. 3 shows the total inelastic cross section in the case of neutrons scattered by a ${ }^{165}$ Ho target. Again, our coupled-channel model describes well the observed experimental data.

We also obtain very good agreement with experimental data for inelastic cross sections for individual excited states. As an example, we show in Fig. 4 the predictions of our model for the angleintegrated inelastic cross section of the first inelastic channel of the target ${ }^{184} \mathrm{~W}$, which is a $2^{+}$state at excitation energy of $111.2 \mathrm{keV}$, as a function of the neutron incident energy. We observe again that we are able to achieve a very good description of the measured data within our coupled channels model. 


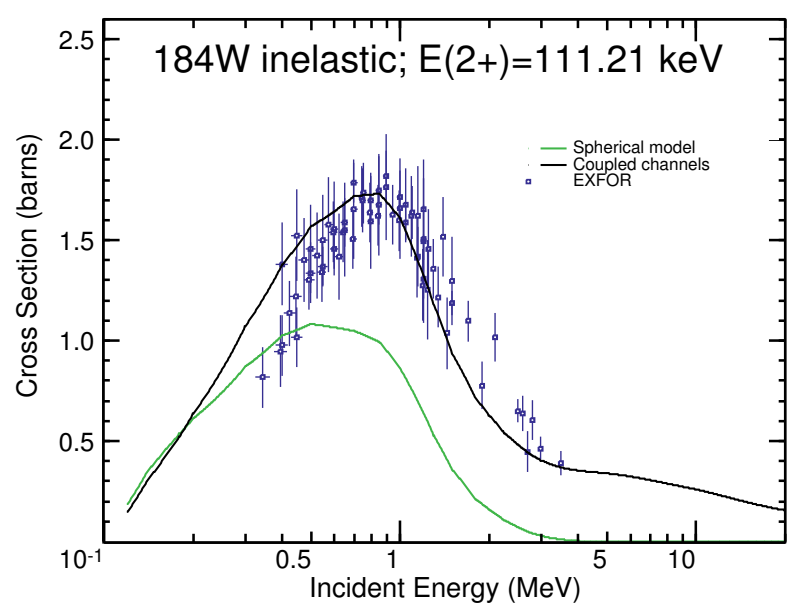

Figure 4. Angle-integrated inelastic cross sections for the $2^{+}$state (excitation energy of $111.2 \mathrm{keV}$ ) of the target ${ }^{184} \mathrm{~W}$, excited on a neutron-induced reaction. The black curve corresponds to coupled-channel calculations within our model, while the green curve indicates, for comparison purposes, the result from a spherical model calculation, using the Hauser-Feshbach model to describe the excitation. The experimental data were taken from EXFOR [11].

\section{Angular Distributions}

A more careful analysis of differential cross-section experimental data proved necessary due to the large amount of angular distribution data available in the literature, and also because some measurements do not contain pure elastic isotopic data. It is quite common for experiments measuring elastic angular distributions for rare-earth nuclei to be unable to separate inelastic contributions due to the low-lying excitation energies of their rotational states. In such cases, measured data correspond actually to "quasi-elastic" angular distributions, and the calculated elastic and inelastic differential cross sections have to added up together accordingly for appropriate comparison. In addition, some experiments were done using the natural form of the element, rather than the isotope-specific one.

For these reasons, application of the coupled-channel model for angular distributions was focused on three elements only: Gadolinium, Holmium, and Tungsten. Those three elements were chosen because the lighter and heavier ones are close to the border of the rare-earth region, while the other is roughly in the middle.

\subsection{Volume Conservation}

When an originally spherical configuration assumes a deformed shape, defined by quadrupole and hexadecupole deformation parameters $\beta_{2}$ and $\beta_{4}$, respectively, the volume and densities are not conserved. In Ref. [23], a method to ensure volume conservation was proposed, corresponding to applying a correction to the reduced radius $R_{0}$, of the form:

$$
R_{0}^{\prime}=R_{0}\left(1-\sum_{\lambda} \beta_{\lambda}^{2} / 4 \pi\right),
$$

where terms of the order of $\beta_{\lambda}^{3}$ and higher have been discarded. Ref. [3] tested the effects of such a correction, showing that it is not negligible and seems to bring the integral and differential cross-section 


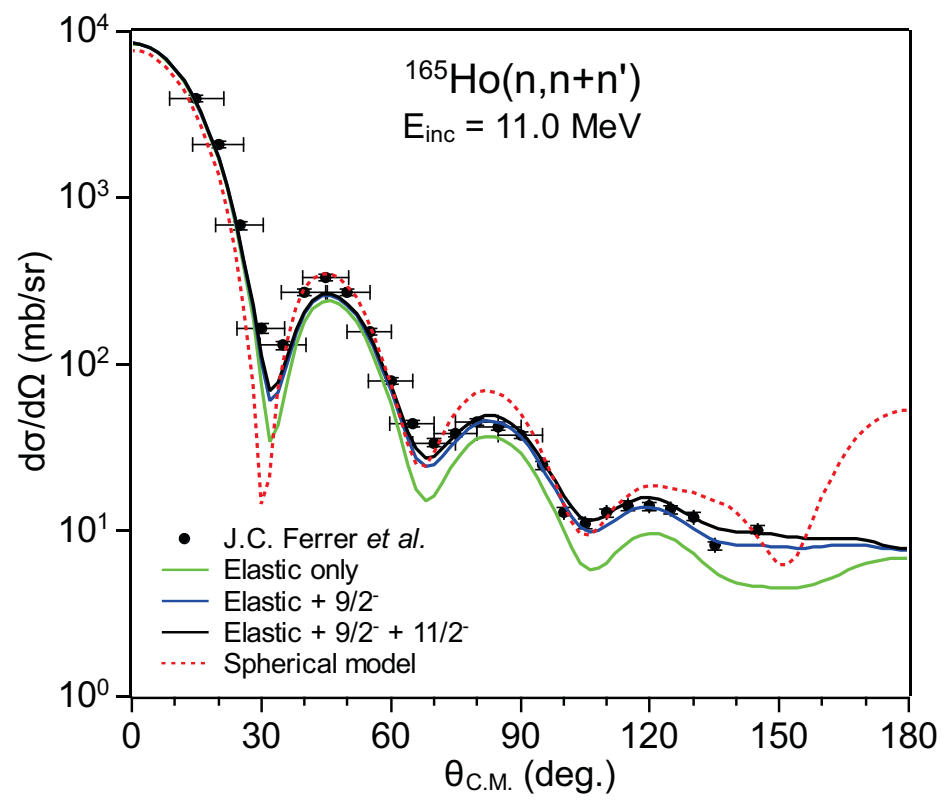

Figure 5. Quasi-elastic angular distribution for the neutron-induced reaction on ${ }^{165} \mathrm{Ho}$. The green curve corresponds to the results for the elastic channel only, obtained within our coupled channels model. The blue and black curves contain, in addition to the elastic differential cross section, contributions from the first, and first and second inelastic channels, respectively. For comparison purposes, we also plot the result from a spherical model calculation, as the dashed-red curve. The experimental data were taken from Ref. [11], corresponding to measurements from Ref. [25].

calculations to a slightly better agreement with the experimental data. Therefore, in the following calculations of angular distributions, we decided to implement the radial corrections calculated from Eq. 2, as it should correspond to a more realistic modeling of the deformed nuclei.

\subsection{Initial results}

In this work we present preliminary results for quasi-elastic differential cross sections for the ${ }^{165} \mathrm{Ho}$ target. In Ref. [12] one can also find preliminary results within the same coupled-channel model of angular distributions for ${ }^{158} \mathrm{Gd}$ and ${ }^{184} \mathrm{~W}$.

The ground state of ${ }^{165} \mathrm{Ho}$ has spin and parity corresponding to $7 / 2^{-}$. As an odd nucleus, its rotational band does not follow the standard $0^{+}, 2^{+}, 4^{+}$, etc., scheme. For coupling purposes, the successive negative-parity states with a difference of spin equal to 1 relative to the ground state, i. e., $7 / 2^{-}, 9 / 2^{-}, 11 / 2^{-}, 13 / 2^{-}$, etc, were considered to belong to the ground state rotational band. Couplechannel calculations were performed coupling up to the $23 / 2^{-}$state. The values adopted for the deformation parameters were $\beta_{2}=0.3$ and $\beta_{4}=-0.020$ [24].

As an example, Fig. 5 presents the predictions of our model when attempting to describe the elastic angular distribution data for ${ }^{165} \mathrm{Ho}$, at the neutron-incident energy of $11 \mathrm{MeV}$, as measured by Ferrer et al. [25]. Actually, a careful analysis of Ref. [25] indicates that in that experiment it was not possible to separate the elastic channel from the inelastic ones. Therefore, the data points in Fig. 5 should contain inelastic contributions. It is seen in Fig. 5 that the predictions of our coupled channels model for 
the elastic angular distribution (green curve) lies consistently below the experimental data. However, when the contribution from the first inelastic state, which is a $9 / 2^{-}$state (excitation energy of $94.7 \mathrm{keV}$ ) is added (blue curve), the coupled channels prediction approaches the observed cross sections. When the second inelastic state $\left(11 / 2^{-}\right.$state lying at $\left.209.8 \mathrm{keV}\right)$ is further added (black curve), we achieve a very good description of the observed quasi-elastic angular distribution. For comparison purposes we plot on the same figure the result obtained from spherical-model calculations, as the dashed red curve.

\section{Conclusion}

In this work, we demonstrated that we can use the spherical Koning-Delaroche optical potential in coupled channels calculations by simply deforming it and making no further modification. We found that we achieved encouraging results in the description of neutron-induced reactions on the rare-earths despite the fact that this potential was not designed to describe reactions on deformed nuclei. We studied the effect of reducing the radius to ensure volume conservation when deforming the original spherical configuration. This correction was found to produce small but significant effects which improved the agreement with experimental data for the cases we tested. With our approach, we described experimental data not only for optical-model observables (such as total cross sections, elastic and inelastic angular distributions), but also for those obtained through compound-nucleus formation (such as total elastic and inelastic, capture cross sections). Our results are consistent with the insight that the scattering is very close to the adiabatic limit as shown in Ref. [1]. Although imperfect, this simple method is a consistent and general first step towards an optical potential capable of fully describing the rare-earth region and fills the need of an optical model potential in this important region.

\section{Acknowledgments}

The work at Brookhaven National Laboratory was sponsored by the Office of Nuclear Physics, Office of Science of the U.S. Department of Energy under Contract No. DE-AC02-98CH10886 with Brookhaven Science Associates, LLC.

\section{References}

[1] F.S. Dietrich, I.J. Thompson, T. Kawano, Phys. Rev. C 85, 044611 (2012)

[2] S. Kunieda, S. Chiba, K. Shibata, A. Ichihara, E.S. Sukhovitskiĩ, Journal of Nuclear Science and Technology 44, 838 (2007), http: //www . tandfonline.com/doi/pdf/10.1080/18811248.2007.9711321

[3] G.P.A. Nobre, A. Palumbo, D. Brown, M. Herman, S. Hoblit, F.S. Dietrich, to be published on Nuclear Data Sheets (2014), http://arxiv.org/abs/1311.0426

[4] A. Koning, J. Delaroche, Nuclear Physics A 713, 231 (2003)

[5] H. Krappe, Annals of Physics 99, 142 (1976)

[6] M. Herman, R. Capote, B. Carlson, P. Obložinský, M. Sin, A. Trkov, H. Wienke, V. Zerkin, Nucl. Data Sheets 108, 2655 (2007)

[7] M. Herman, R. Capote, M. Sin, A. Trkov, B.V. Carlson, C.M.M. P Obložinský, H. Wienke, S. Hoblit, Y.S. Cho, G.P.A. Nobre et al., Tech. Rep. INDC(NDS)-0642, BNL-101378-2013 (2013)

[8] J. Raynal, Tech. Rep. SMR-9/8, IAEA (1970) 
[9] J. Raynal, Optical model and coupled-channels calculations in nuclear physics, in Computing as a language of physics. ICTP International Seminar Course (IAEA, ICTP, Trieste, Italy, 1972), p. 281

[10] S. Raman, C.W. Nestor, Jr., P. Tikkanen, Atomic Data and Nuclear Data Tables 78, 1 (2001)

[11] N. Otuka, E. Dupont, V. Semkova et al., to be published on Nuclear Data Sheets (2014)

[12] G.P.A. Nobre, F.S. Dietrich, M. Herman, A. Palumbo, S. Hoblit, D. Brown, Towards an optical potential for rare-earths through coupled channels, in XXXVI Reunião de Trabalho de Física Nuclear no Brasil (XXXVI Brazilian Workshop on Nuclear Physics) (Maresias, São Paulo, Brazil, to be published on AIP Conference Proceeding Series), http://arxiv.org/abs/1311.1735

[13] A. D’Arrigo, G. Giardina, M. Herman, A.V. Ignatyuk, A. Taccone, J. Phys. G20, 305 (1994)

[14] V.A. Plujko, Acta Phys. Pol. B 31, 435 (2000)

[15] V.A. Plujko, in $9^{\text {th }}$ International Conference for Nuclear Reaction Mechanisms, edited by E. Gadioli (Universita degli Studi di Milano, Varenna, Italy, 2000), Vol. Suppl. 115, p. 113

[16] V.A. Plujko, S.N. Ezhov, M.O. Kavatsyuk, in International Conference on Nuclear Data for Science and Technology, edited by K. Shibata (Atomic Energy Society of Japan, Tsukuba, Japan, 2001), Vol. 1, p. 811

[17] H.M. Hofmann, J. Richert, J.W. Tepel, H.A. Weidenmüller, Ann. Phys. 90, 403 (1975)

[18] H.M. Hofmann, T. Mertelmeier, M. Herman, J.W. Tepel, Z. Physik A 297, 153 (1980)

[19] J.J. Griffin, Phys. Rev. Lett. 17, 478 (1966)

[20] C.K. Cline, M. Blann, Nucl. Phys. A 172, 225 (1971)

[21] C.K. Cline, Nucl. Phys. A193, 417 (1972)

[22] I. Ribanský, P. Obložinský, E. Bětak, Nucl. Phys. A205, 545 (1973)

[23] J. Bang, J. Vaagen, Zeitschrift für Physik A Atoms and Nuclei 297, 223 (1980)

[24] A.B. Smith, Annals of Nuclear Energy 28, 1745 (2001)

[25] J. Ferrer, J. Carlson, J. Rapaport, Nuclear Physics A 275, 325 (1977) 